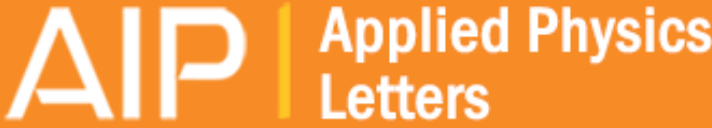

\section{Terahertz electro-absorption effect enabling femtosecond all-optical switching in semiconductor quantum dots}

M. C. Hoffmann, B. S. Monozon, D. Livshits, E. U. Rafailov, and D. Turchinovich

Citation: Applied Physics Letters 97, 231108 (2010); doi: 10.1063/1.3515909

View online: http://dx.doi.org/10.1063/1.3515909

View Table of Contents: http://scitation.aip.org/content/aip/journal/apl/97/23?ver=pdfcov

Published by the AIP Publishing

\section{Articles you may be interested in}

Vertical-geometry all-optical switches based on InAs/GaAs quantum dots in a cavity

Appl. Phys. Lett. 95, 021109 (2009); 10.1063/1.3180704

Characteristics of $1.3 \mu \mathrm{m} \ln A s / \mathrm{lnGaAs} / \mathrm{GaAs}$ quantum dot electroabsorption modulator

Appl. Phys. Lett. 94, 143108 (2009); 10.1063/1.3119186

Electroabsorption and electrorefraction in In As/Ga As and In As/In P quantum dots

J. Appl. Phys. 99, 054501 (2006); 10.1063/1.2175469

Terahertz-frequency electronic coupling in vertically coupled quantum dots

Appl. Phys. Lett. 77, 4356 (2000); 10.1063/1.1334912

The electro-optic properties of interdiffused InGaAs/InP quantum well structures

J. Appl. Phys. 88, 3418 (2000); 10.1063/1.1285840

Frustrated by

old technology?

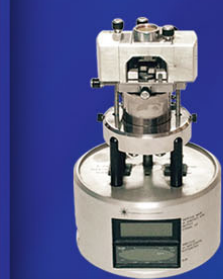

Is your AFM dead

and can't be repaired?

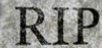

My Old AFM 1994-2015
Sick of bad customer support?

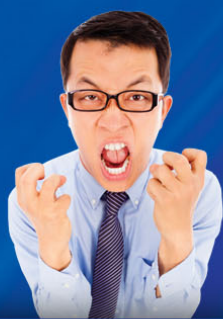

It is time to upgrade your AFM

Minimum \$20,000 trade-in discount

for purchases before August 31st

Asylum Research is today's technology leader in AFM 


\title{
Terahertz electro-absorption effect enabling femtosecond all-optical switching in semiconductor quantum dots
}

\author{
M. C. Hoffmann, ${ }^{1, a)}$ B. S. Monozon, ${ }^{2}$ D. Livshits, ${ }^{3}$ E. U. Rafailov, ${ }^{4}$ and D. Turchinovich ${ }^{5, b)}$ \\ ${ }^{1}$ Max Planck Research Department for Structural Dynamics, University of Hamburg, CFEL, 22607 \\ Hamburg, Germany \\ ${ }^{2}$ Department of Physics, State Marine Technical University, Lotsmanskaya 3, 190008 St. Petersburg, Russia \\ ${ }^{3}$ Innolume GmbH, Konrad-Adenauer-Allee 11, 44263 Dortmund, Germany \\ ${ }^{4}$ School of Engineering, Physics, and Mathematics, University of Dundee, Dundee DD1 4HN, \\ United Kingdom \\ ${ }^{5}$ DTU Fotonik - Department of Photonics Engineering, Technical University of Denmark, DK-2800 Kgs. \\ Lyngby, Denmark
}

(Received 24 September 2010; accepted 25 October 2010; published online 8 December 2010)

\begin{abstract}
We demonstrate an instantaneous all-optical manipulation of optical absorption in InGaAs/GaAs quantum dots (QDs) via an electro-absorption effect induced by the electric field of an incident free-space terahertz signal. A terahertz signal with the full bandwidth of $3 \mathrm{THz}$ was directly encoded onto an optical signal probing the absorption in QDs, resulting in the encoded temporal features as fast as $460 \mathrm{fs}$. The instantaneous nature of this effect enables femtosecond all-optical switching at very high repetition rates, suggesting applications in terahertz-range wireless communication systems with data rates of at least 0.5 Tbit/s. (c) 2010 American Institute of Physics. [doi:10.1063/1.3515909]
\end{abstract}

Modern optical communication systems are reaching Tbit/s data rates in single serial communication channels. ${ }^{1}$ Time and bandwidth considerations require that optical signals with femtosecond duration and terahertz (THz) bandwidth must be used as data symbols in such systems. In order to implement a wireless (sub)Tbit/s system, such as e.g., radio-over-fiber (RoF) system, the signal frequency must be in the THz range, ${ }^{2}$ and femtosecond, single-cycle THz pulses should be used as data symbols. The means to encode such ultrafast $\mathrm{THz}$ signals onto an optical carrier at (sub)THz repetition rate should also be provided.

It was recently demonstrated that a continuous-wave (cw) $\mathrm{THz}$ signal originating from a cryogenically cooled quantum-cascade laser (QCL) can be encoded onto a cw optical carrier propagating through the waveguide comprising the QCL by THz-induced phase modulation via nonlinear-optical frequency mixing. As a result, the THzspaced sidebands were observed in the spectrum of $\mathrm{cw}$ optical carrier. ${ }^{3}$

In this work we demonstrate femtosecond all-optical switching of optical absorption in InGaAs/GaAs quantum dots (QDs) via the instantaneous electro-absorption effect induced by the electric field of an incident $\mathrm{THz}$ pulse. As a result, we achieved a direct all-optical encoding of a freespace, ultrafast, high-bandwidth, and high repetition rate $\mathrm{THz}$ signal onto an optical signal probing the absorption in the QDs.

The electro-absorption effect in a QD, a quantumconfined Stark effect ${ }^{4}$ (QCSE), arises from the tilt in the confinement potential of a semiconductor nanostructure in the applied electric field $E$ and manifests itself in two ways: (i) in a decrease of the optical transition energy between electron and hole states (Stark shift) and (ii) in a spatial separation of the envelope wavefunctions of electrons $\psi_{e}$ and holes $\psi_{h}$ in the presence of the electric field, which leads to a

\footnotetext{
${ }^{a)}$ Electronic mail: matthias.c.hoffmann@desy.de.

${ }^{b)}$ Electronic mail: dmtu@fotonik.dtu.dk.
}

smaller wavefunction overlap integral $M(E)=\left\langle\psi_{e} \mid \psi_{h}\right\rangle$. The value of $|M(E)|^{2}$ dictates the optical transition probability, and the optical absorption coefficient is directly proportional to it. Thus, the optical absorption can be manipulated by the applied electric field. In the case of a spatially symmetric confinement potential at $E=0$, the strength of the electroabsorption modulation effect only depends on the absolute value of the electric field and not on its sign.

Instead of applying the modulating electric field to the whole QD structure via the hard-wired electrodes, which would slow down the modulation rate due to a large RCconstant involved, we modulate the confinement potential of the QDs (which are the smallest possible semiconductor elements with a negligible RC constant) directly by the electric field of single-cycle $\mathrm{THz}$ pulses directed at the QD sample at normal incidence angle.

The sample used in our experiments was an InGaAs/ GaAs QD-based semiconductor saturable absorber mirror (SESAM), comprising of a broadband $\mathrm{GaAs} / \mathrm{Al}_{0.9} \mathrm{Ga}_{0.1} \mathrm{As}$ distributed Bragg reflector (DBR), and a QD absorber layer featuring 80 layers of submonolayer-grown $\mathrm{In}_{0.5} \mathrm{Ga}_{0.5} \mathrm{As}$ / GaAs QDs with the diameter of around $10 \mathrm{~nm}$ and the height of around $7 \mathrm{~nm}$, alternated by the GaAs barriers of 10-14 nm thickness. Eight of these barriers were grown at low temperature (LT) in order to provide for the fast SESAM partial recovery with the exponential time constant of 4.3 ps for mode-locked laser applications, unrelated to this work. The QD absorber layer and the DBR were grown on a $(450 \pm 25) \mu \mathrm{m}$ thick, $\langle 100\rangle$-oriented semi-insulating GaAs substrate. The small-signal optical reflectivity spectra of the whole QD sample, and of a bare DBR measured prior to the growth of the QD absorber layer, are shown in Fig. 1. The dip in the reflectivity of a QD sample positioned around $1040 \mathrm{~nm}$ is due to ground-state absorption in the QDs. The absence of in-plane conductivity due to the carriers occupying the QD ground state and a 35 ps long carrier thermalization time were observed in a similarly grown structure, dem- 


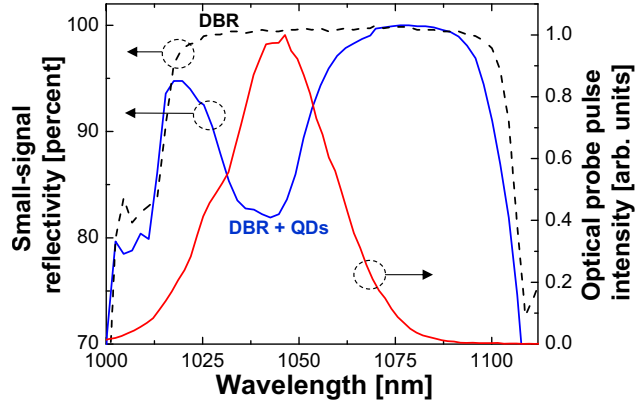

FIG. 1. (Color online) Small-signal reflectivity spectrum of the whole QD sample (solid line), a bare DBR (dashed line), and the intensity spectrum of the probe laser pulse at QD ground state resonance at $1040 \mathrm{~nm}$.

onstrating the strong carrier confinement to the QDs at room temperature. ${ }^{5}$

Single-cycle $\mathrm{THz}$ pulses were generated by optical rectification of $800-\mathrm{nm}, 80 \mathrm{fs}$ long Ti:sapphire laser pulses of $2 \mathrm{~mJ}$ energy in a lithium niobate crystal at $1 \mathrm{kHz}$ repetition rate using the tilted pulse-front technique, ${ }^{6}$ yielding $\mathrm{THz}$ pulse energies of $2 \mu \mathrm{J}$. The $\mathrm{THz}$ pulses were characterized in the time-domain using a standard free-space electro-optic sampling in a combined "active-passive"" $0.2 \mathrm{~mm}\langle 110\rangle-2 \mathrm{~mm}\langle 100\rangle$ undoped $\mathrm{GaP}$ crystal, and showed a spectrum covering the range of $0.2-3 \mathrm{THz}$. The peak electric field of the strongest $\mathrm{THz}$ transient was estimated $^{8}$ to be $220 \mathrm{kV} / \mathrm{cm}$ in air. The $\mathrm{THz}$ waveform and its amplitude spectrum are shown in Fig. 2(a). A weak optical probe pulse around the wavelength of $1040 \mathrm{~nm}$, coinciding with the ground state absorption feature in the QDs (see Fig. 1), was produced by conversion of part of the $800 \mathrm{~nm}$ laser output, using an optical parametric amplifier.

We performed a THz pump - optical probe experiment on our sample, where the probe pulse reflectivity off the QD
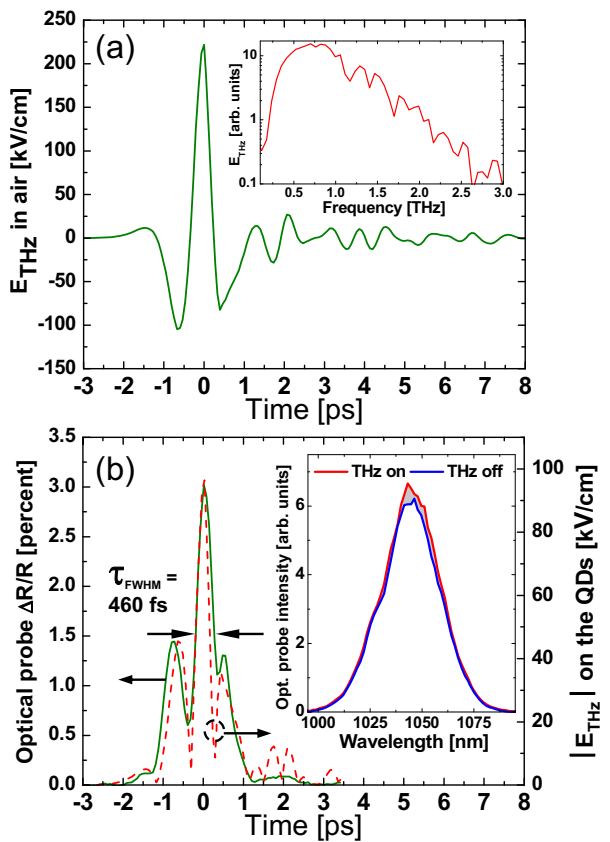

FIG. 2. (Color online) (a) Electric field in free-space as a function of time of the $\mathrm{THz}$ pulse with peak field strength of $220 \mathrm{kV} / \mathrm{cm}$. Inset: its amplitude frequency spectrum. (b) Solid line: $\Delta R / R$ of the probe signal at $1040 \mathrm{~nm}$ in the QD sample, under influence of the incident THz pulse from (a). Dashed line: absolute value of the electric field in the THz pulse from (a) experienced by the QDs. Inset: intensity spectrum of the optical probe signal with and without peak electric field of the $\mathrm{THz}$ signal from (a) on the QDs. structure was modulated by the incident $\mathrm{THz}$ pulse. We measured the absolute value and the sign of the change in the optical probe reflectivity as a function of time-delay between the optical probe and $\mathrm{THz}$ pump pulses, using the photodiodes in a balanced detection arrangement. The angles of incidence on the sample (with respect to the normal) of the pump THz and probe optical beams were $0^{\circ}$ and $10^{\circ}$, respectively. The optical probe signal interacted with the QDs twice: on the way to and from the DBR. However, the total thickness of the QD absorber layer, including GaAs barriers, and spacer and cladding layers, is only about $1.2 \mu \mathrm{m}$, thus the interaction of both the $\mathrm{THz}$ pulse and the probe pulse with the QDs can be viewed as point interactions. All our measurements were performed at room temperature and in ambient atmospheric conditions. The dips in the amplitude spectrum of the THz signal in Fig. 2(a) are absorption lines of atmospheric water vapor.

In Fig. 2(b) our main result, a modulation of the reflectivity of the optical probe $\Delta R / R$, is shown, along with the absolute value of the electric field of the THz pulse $\left|E_{\mathrm{THz}}\right|$, experienced by the QDs. The latter was calculated taking into account the $\mathrm{THz}$ field transmission coefficient at the sample GaAs interface of 0.435 . The observed $\Delta R / R$ of the optical signal follows the shape of $\left|E_{\mathrm{THz}}\right|$ in a temporally coherent manner, reproduces most of the features of the $\mathrm{THz}$ signal, and does not exhibit exponential-like decay features attributed to a relaxation process of any sort. The $\mathrm{THz}$ pulse features as fast as $460 \mathrm{fs}$ at full width at half maximum are encoded all-optically onto the probe signal transmitted through the QDs. Obviously, these femtosecond-scale features cannot originate from a carrier depletion mechanism in QDs related to carrier trapping from the QDs onto lattice defects in the LT-GaAs barriers. This process with a decay time constant of $4.3 \mathrm{ps}$ is nearly ten times slower than the observed fast modulations. The maximum observed $\Delta R / R$ value was about $3 \%$, at the peak $\mathrm{THz}$ electric field on the QDs of $96.5 \mathrm{kV} / \mathrm{cm}$. Following Ref. 9 we calculated the small-signal loss $S$ experienced by the optical probe with the spectrum $I(\lambda)$ in the QDs as $S=\int_{\lambda} I(\lambda)\left[R_{\mathrm{DBR}}(\lambda)\right.$ $\left.-R_{\mathrm{QD}}(\lambda)\right] d \lambda / \int_{\lambda} I(\lambda) d \lambda=10.3 \%$, where $R_{\mathrm{DBR}, \mathrm{QD}}$ are the reflectivity spectra of the bare DBR and of the whole QD sample (see Fig. 1). We were thus able to reach a $30 \%$ modulation of the QD absorption experienced by the optical probe. This is comparable to the modulation depth observed in resonantly probed quantum wells (QWs) at similar static bias field strengths. ${ }^{9}$ When the probe wavelength was de-tuned from the QD ground state resonance, the magnitude of $\Delta R / R$ signal decreased by an order of magnitude, proving that the observed modulation is indeed due to the interaction of the QDs with the THz field. None, or very weak dependency of the electro-absorption modulation strength on the polarizations of $\mathrm{THz}$ and optical signals, respectively, was observed, as is indeed expected from the three-dimensional QD geometry.

The sign of the observed reflectivity change $\Delta R / R$ is positive, corresponding to a decrease in optical absorption at the probe wavelength in the presence of $\mathrm{THz}$ electric field. This positive sign of $\Delta R / R$ suggests that both manifestations of the QCSE may play a role: overall optical absorption quenching; and Stark shift of the QD absorption spectrum toward longer wavelengths, i.e., out of the probe pulse spectrum. The inset of Fig. 2(b) shows the spectrum of the 

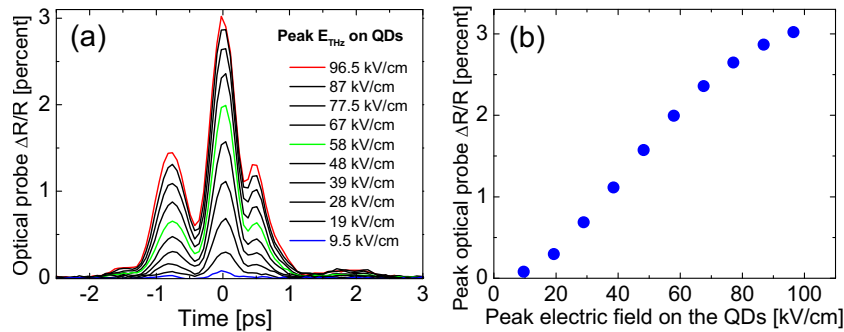

FIG. 3. (Color online) (a) $\Delta R / R$ of the optical probe signal in the QD sample, under influence of the incident THz pulses with variable peak field strength. (b) Peak values of $\Delta R / R$ of the signals from (a), as a function of peak $\mathrm{THz}$ field on the QDs.

optical probe pulse in the case when the maximum of the THz pulse was in temporal overlap with the probe pulse, and when the $\mathrm{THz}$ pulse was blocked. The change in the probe pulse amplitude is more or less spectrally homogeneous, suggesting that the absorption quenching in the QDs is likely to be a dominating electro-absorption modulation effect here.

Figure 3(a) shows the optical probe modulation signals $\Delta R / R$ for different $\mathrm{THz}$ field strengths. The $\mathrm{THz}$ electric field was controlled using a pair of wire-grid polarizers. ${ }^{10} \mathrm{In}$ Fig. 3(b) the peak value of $\Delta R / R$ as a function of the peak THz electric field experienced by the QDs is shown, demonstrating a pronounced nonlinear scaling with the electric field, typical for the QCSE. ${ }^{4}$

We note that THz-induced electro-absorption modulation in semiconductors has been studied in the past theoretically. ${ }^{11}$ Recently, it was observed at the exciton resonances in QWs ${ }^{12}$ and carbon nanotubes. ${ }^{13}$ The excitons were polarized by the $\mathrm{THz}$ field in the plane of QWs or along the nanotube long dimension. Stronger THz fields lead to ionization of excitons in QWs. ${ }^{12}$ The THz modulation rate limit of around $1 \mathrm{THz}$ was observed in Ref. 13, and was related to the exciton dephasing time (also see discussion in Ref. 12). In the case of QDs, the modulation rate should probably be limited by the $\mathrm{THz}$ frequencies high enough to excite the intraband transitions in the QDs, i.e., by the frequencies of $10 \mathrm{THz}$ and higher.

Finally, in Fig. 4 we show the modulation of optical absorption in the QDs induced by a train of THz pulses incident at high repetition rate. This $\mathrm{THz}$ pulse train originated from multiple reflections of a single $\mathrm{THz}$ pulse within the sample, which was attached to a metal mirror with the back

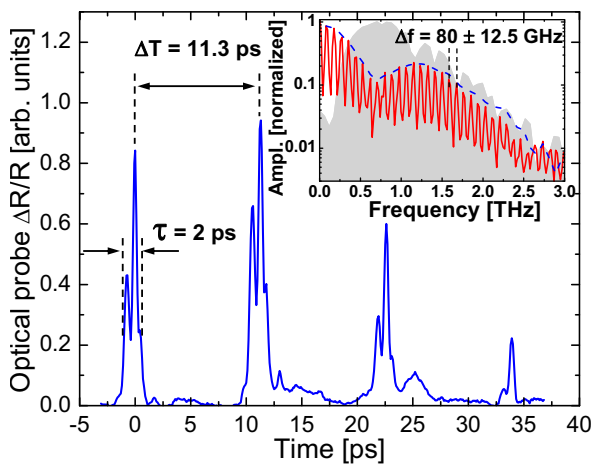

FIG. 4. (Color online) $\Delta R / R$ of the optical probe signal in the QDs, provided by the multiple reflections of a single $\mathrm{THz}$ pulse in the QD sample. Inset: amplitude Fourier spectra of the isolated first $\Delta R / R$ modulation pulse around 0 ps (dashed line), and of the full multipulse sequence. Background: amplitude spectrum of a single $\mathrm{THz}$ pulse. side of its substrate. The individual pulses of $\Delta R / R$ have a temporal width of around 2 ps. Importantly, the contrast between the peak $\Delta R / R$ and the background is rather high. A certain reshaping of $\Delta R / R$ pulses in the sequence is due to the dispersion and scattering of the $\mathrm{THz}$ pulse experiencing multiple reflections within the sample. The interpulse interval of 11.3 ps (i.e., repetition rate of $88 \mathrm{GHz}$ ) corresponding to the round-trip time of the $\mathrm{THz}$ pulse within the sample, shows the possibility of $88 \mathrm{Gbit} / \mathrm{s}$ data rate using this electroabsorption modulation scheme. The ratio of interpulse interval to the individual pulse duration $11.3 \mathrm{ps} / 2 \mathrm{ps}=5.65$ suggests that the demonstrated data rate can be scaled accordingly, and crosstalk-free data rates of $88 \mathrm{Gbit} / \mathrm{s} \times 5.65$ $=0.5 \mathrm{Tbit} / \mathrm{s}$ can be achieved .

In the inset of Fig. 4, the amplitude Fourier spectra of the isolated $\Delta R / R$ pulse and of the whole multipulse sequence are shown, with the amplitude spectrum of the single $\mathrm{THz}$ pulse in the background. It can be clearly seen that the full bandwidth of the $\mathrm{THz}$ pulse of $3 \mathrm{THz}$ is encoded onto the optical probe signal, and the repetition rate is also reflected in the spectrum. These Fourier spectra are analogous to the $\mathrm{THz}$ sidebands observed in work. ${ }^{3}$

In conclusion, we have demonstrated instantaneous and polarization-independent electro-absorption modulation in QDs on the order of several percent, by the broadband $\mathrm{THz}$ pulses incident from a free-space. This effect may find its applications in future (sub)Tbit/s RoF systems, as well as in general-purpose coherent THz-to-optical detectors/encoders. Optimization of interaction of QDs with $\mathrm{THz}$ field ${ }^{14,15}$ can lead to a THz electro-absorption modulator controlled by the low switching fields.

We are grateful to Max Planck Society and EU FP7 Programme (FAST-DOT, Grant No. 224338) for partial financial support, and to A. Cavalleri (University of Hamburg), A.-P. Jauho (DTU Nanotech), and K. Yvind and J. M. Hvam (DTU Fotonik) for valuable discussions.

${ }^{1}$ H. C. H. Mulvad, M. Galili, L. K. Oxenløwe, H. Hu, A. T. Clausen, J. B. Jensen, C. Peucheret, and P. Jeppesen, Opt. Express 18, 1438 (2010).

${ }^{2}$ J. Federici and L. Moeller, J. Appl. Phys. 107, 111101 (2010).

${ }^{3}$ S. S. Dhillon, C. Sirtori, J. Alton, S. Barbieri, A. De Rossi, H. E. Beere, and D. A. Ritchie, Nat. Photonics 1, 411 (2007).

${ }^{4}$ D. A. B. Miller, D. S. Chemla, T. C. Damen, A. C. Gossard, W. Wiegmann, T. H. Wood, and C. A. Burrus, Phys. Rev. Lett. 53, 2173 (1984).

${ }^{5}$ H. P. Porte, P. U. Jepsen, N. Daghestani, E. U. Rafailov, and D. Turchinovich, Appl. Phys. Lett. 94, 262104 (2009).

${ }^{6}$ K.-L. Yeh, M. C. Hoffmann, J. Hebling, and K. A. Nelson, Appl. Phys. Lett. 90, 171121 (2007).

${ }^{7}$ D. Turchinovich and J. I. Dijkhuis, Opt. Commun. 270, 96 (2007).

${ }^{8}$ F. Blanchard, L. Razzari, H. C. Bandulet, G. Sharma, R. Morandotti, J. C. Kieffer, T. Ozaki, M. Reid, H. F. Tiedje, H. K. Haugen, and F. A. Hegmann, Opt. Express 15, 13212 (2007).

${ }^{9}$ X. Liu, E. U. Rafailov, D. Livshits, and D. Turchinovich, Appl. Phys. Lett. 97, 051103 (2010).

${ }^{10}$ M. C. Hoffmann and D. Turchinovich, Appl. Phys. Lett. 96, 151110 (2010).

${ }^{11}$ K. Johnsen and A.-P. Jauho, Phys. Rev. B 57, 8860 (1998).

${ }^{12}$ H. Hirori, M. Nagai, and K. Tanaka, Phys. Rev. B 81, 081305(R) (2010).

${ }^{13}$ T. Ogawa, S. Watanabe, N. Minami, and R. Shimano, Appl. Phys. Lett. 97, 041111 (2010).

${ }^{14}$ M. A. Seo, H. R. Park, S. M. Koo, D. J. Park, J. H. Kang, O. K. Suwal, S S. Choi, P. C. M. Planken, G. S. Park, N. K. Park, Q. H. Park, and D. S. Kim, Nat. Photonics 3, 152 (2009).

${ }^{15}$ D. Turchinovich, patent application PCT/DK2010/050200, 05 August (2009). 